\title{
Avaliação da qualidade de luvas de látex utilizadas em procedimentos odontológicos
}

\author{
Quality evaluation of latex gloves used in dental procedures
}

\author{
Júlio Neto Souto Batista \\ Maria Dara do Rego Alves Rodrigues \\ Ravenna Melo Vieira Gomes \\ Faculdade de Odontologia, Universidade Federal do Piauí, Teresina, Piauí, Brasil \\ Caroline de Deus Tupinambá Rodrigues \\ Alessandro Ribeiro Gonçalves \\ Departamento de Odontologia Restauradora, Faculdade de Odontologia, Universidade Federal do Piauí, Teresina, Piauí, Brasil \\ - Os autores declaram que não há conflito de interesse.
}

\section{Resumo}

Objetivo: 0 trabalho verificou a qualidade de luvas de látex de procedimento de cinco marcas comerciais utilizadas na prática clínica. Material e Métodos: Foram realizados os testes de Inspeção Visual (IV) e de Integridade (TI) em luvas de procedimento a fim de detectar possíveis defeitos de fabricação. Foram avaliadas 1007 luvas de procedimento (duas caixas tamanho M de cada fabricante). Resultados: Do total, 3,87\% apresentaram problemas no teste IV e 1,96\% no TI. A marca SATARI 8 obteve melhor desempenho no teste IV, enquanto a SUPERMAX® o pior. A marca DESCARPACK® obteve o melhor desempenho no TI e a EMBRAMAC@ o pior. Conclusão: No quesito hermeticidade, em comparação com o nivel de qualidade aceitável, imposto pelo INMETRO, as marcas EMBRAMAC $\circledast$ e LEMGRUBER $®$ foram reprovadas.

Palavras-chave: exposição a agentes biológicos; controle de infecções; saúde do trabalhador.

\section{ABstract}

Objective: The objective of this study was to verify the quality of latex procedure gloves of 5 trademarks used in clinical practice. Material and Methods: Visual Inspection (VI) and Integrity (IT) tests were performed in procedure gloves in order to detect possible manufacture defects. A number of 1007 procedure gloves (2 packages $\mathrm{M}$ size of each manufacturer) were evaluated. Results: In general, 3,87\% showed defects in the VI test and 1,98\% in the IT. The brand SATARI had the best performance in the VI test, whereas the brand SUPERMAX® had the worst. Conclusion: The make DESCARPACK® had the best performance in the IT and the brand EMBRAMAC® had the worst. In the hermeticity aspect, in comparison to the acceptable quality level imposed by INMETRO, the brands EMBRAMAC@ and LEMGRUBER ${ }^{8}$ were reproved. Keywords: exposure to biological agents; infection control; occupational health.

\section{Introdução}

A manipulação química, biológica e física de produtos pode causar sérios danos à saúde do trabalhador. Estes riscos de agravos podem ser provenientes de diferentes formas, sendo classificados pela existência de agentes biológicos, químicos, físicos, ergonômicos e mecânicos. Dessa forma, há a necessidade de estabelecer procedimentos e ações de biossegurança para garantir a saúde do trabalhador. ${ }^{1}$ A Odontologia lidera o número de acidentes envolvendo material biológico, devido ao fato de que estes profissionais lidam, durante toda sua jornada de trabalho, com materiais perfurocortantes, instrumentos rotatórios que produzem aerossol e fluidos corporais. ${ }^{2}$

Samaranayake, Sheutz e Cottone ${ }^{3}$ definiram infecção cruzada como sendo a transmissão de agentes infecciosos entre pacientes e equipe, dentro de um ambiente clínico, cuja transmissão pode resultar do contato pessoa-pessoa ou através de objetos contaminados, que são denominados agentes. O principal meio de prevenção contra a transmissão dessas doenças é o uso adequado dos equipamentos de proteção individuais (EPI). ${ }^{4}$

Qualquer procedimento odontológico, desde um simples exame clínico até cirurgias complexas, deve seguir normas de biossegurança e o cirurgião-dentista e sua equipe devem conhecê-las e, obrigatoriamente, aplicá-las dentro das normas estabelecidas pelos órgãos oficiais responsáveis. ${ }^{5}$

As luvas cirúrgicas e de procedimentos são barreiras fundamentais na prevenção contra infecções como hepatite B e HIV, desde que a integridade dessas luvas, durante a realização dos procedimentos odontológicos, seja mantida. Caso contrário, existe o risco de infecção cruzada. ${ }^{6}$ As luvas possuem três funções principais: reduzem a possibilidade de o profissional se infectar com patógenos presentes no sangue e/ou na saliva dos pacientes, diminuem as chances de o cirurgião-dentista transmitir micro-organismos da microbiota própria de suas mãos para os pacientes, reduzem as possibilidades da transmissão de micro-organismos orais de um paciente para outro. ${ }^{7,8}$

Nesse contexto, surgiu uma preocupação com a possibilidade de as luvas não servirem como barreiras adequadas à passagem de micro-organismos, principalmente aos vírus, ${ }^{6}$ já que, para que elas exerçam adequadamente sua função, é necessário que possuam boa qualidade e não apresentem solução de continuidade que permitam a difusão desses patógenos. ${ }^{8}$ Portanto justifica-se a necessidade de estudos que avaliem a qualidade das luvas de procedimento utilizadas na clínica odontológica a fim de assegurar a devida proteção tanto para os profissionais que as utilizam como para os pacientes.

Assim, essa pesquisa teve como objetivo verificar a qualidade de luvas de látex, do tipo procedimento, de diferentes marcas comerciais utilizadas na prática clínica. 


\section{Material e Métodos}

O delineamento da pesquisa foi do tipo experimental quantitativo e visou demonstrar o panorama real das luvas de látex testadas, com total imparcialidade em relação a qualquer marca específica. Foram realizados testes com cinco marcas comerciais de luvas de procedimento comumente utilizadas na prática odontológica a fim de detectar possíveis defeitos oriundos de fábrica que comprometam a qualidade destes produtos.

A escolha das marcas de luvas foi a partir de uma pesquisa informal em lojas de materiais dentários da cidade de Teresina (Piauí). As marcas mais utilizadas pelos profissionais da Odontologia da região foram selecionadas para serem testadas.

Foram realizados dois testes: Teste IV - inspeção visual e Teste TI - teste de integridade, ambos adaptados dos trabalhos de Pitten; Herdemann e Kramer, Korniewicz; Garzon e Plitcha, Korniewicz et. al, Machado et al., Phalen e Wong. ${ }^{9-13}$ Foram utilizadas um total de 1007 luvas de procedimento para a realização dos dois testes (IV e TI). Cada marca comercial foi representada por duas caixas (cada caixa com aproximadamente 100 luvas ambidestras tamanho M) de mesmo lote e validade. As luvas foram divididas em grupos de acordo com as marcas comerciais, sendo cinco grupos-teste.

As cinco marcas de luvas estudadas foram: Embramac $^{\bullet}$ (LOTE: 1732 349; FAB: 06/2012; VAL: 5 anos) compondo o Grupo Teste 1 (GT1); Descarpack ${ }^{\circledast}$ (LOTE: 078ME; FAB: 02/2012; VAL: 5 anos) constituindo o Grupo Teste 2 (GT2); Satari ${ }^{\circledR}$ (LOTE: 578614300412 0426; FAB: 04/2012; VAL: 3 anos) o Grupo Teste 3 (GT3); Lemgruber ${ }^{\circledast}$ (LOTE: PR 14084; FAB: 08/2012; VAL: 3 anos) o Grupo Teste 4 (GT4) e Supermax (LOTE: 6220 7914; FAB: 07/2012; VAL: 5 anos) compôs o Grupo Teste 5 (GT5).

Inicialmente, contaram-se quantas luvas continham em cada caixa. O teste IV teve como objetivo detectar presença de defeitos estruturais nas luvas, tais como manchas, regiões aderidas, rasgos ou furos visivelmente detectáveis. Os defeitos detectados foram anotados em uma ficha de coleta de dados em relação à prevalência e a localização (região palmar, região dorsal, região dos dedos). Quando observado a presença de dois ou mais defeitos estruturais em uma mesma luva, considerava-se apenas o mais evidente.

Era considerada mancha, qualquer escurecimento ou esbranquiçamento visível a olho nu; e área aderida qualquer aglomerado ou protuberância observada na luva. Considerou-se furo ou rasgo visível qualquer interrupção da continuidade do látex com formação de orifício na luva (Figura 1).

Por último foi realizado o teste TI, no qual cada luva de procedimento foi preenchida com $500 \mathrm{ml}$ de um líquido com corante evidenciador à temperatura ambiente e aprisionada pelo punho para verificação de vazamentos. ${ }^{6,13,14}$

As luvas, após o preenchimento, foram secas com toalhas de papel absorvente na sua superfície externa e suspensas individualmente em suporte específico, sendo amarradas em suas bases, por 2 horas, a uma distância de 1 metro da bancada. Sobre a bancada foram colocadas folhas de papel absorvente, com o intuito de observar possíveis vazamentos, através de manchas no papel (Figura 2). Cada grupo foi observado em intervalos 30 minutos e registrada a presença de manchas no papel como sinal de vazamentos.

O líquido escapado foi analisado utilizando-se os seguintes escores: não ocorreu vazamento- escore 0; quantidade pequena de vazamento, por gotejamento de uma única perfuração- escore 1; grande quantidade de vazamento por escapamento em jato de uma única perfuração, ou por gotejamento de múltiplas perfurações- escore 2 (Figura 3). Foram anotadas, também, as localizações das perfurações (região palmar, região dorsal, região dos dedos).

Após os testes, calculou-se a porcentagem de luvas aprovadas para o uso (luvas que ao final do Teste TI permaneciam com escore 0 ).

A presença de quaisquer defeitos estruturais no primeiro teste eliminava a participação da luva danificada no teste sucessor.

Os testes foram realizados por dois avaliadores que passaram previamente por calibragem intra e interexaminadores (índice de kappa igual a 0,9). Antes da execução da pesquisa propriamente dita, foi realizado um estudo piloto com $10 \%$ da amostra das luvas para a detecção de possíveis falhas na metodologia e proceder aos ajustes necessários.

Os dados foram armazenados e processados no programa BioEstat 2.0. Os resultados foram submetidos ao Teste Binomial para duas proporções em nível de 5,0\% de significância $(\mathrm{p}=0,05)$.

\section{Resultados}

Em nenhuma das caixas de luvas dos grupos de teste foi encontrada uma quantidade de luvas inferior a 100. Os resultados encontrados estão descritos nas tabelas 1, 2 e 3 . Das 1007 luvas analisadas 39 (3,87\%) apresentaram defeitos estruturais visivelmente detectáveis (manchas, regiões aderidas, rasgos ou furos) (Tabela 1); sendo a marca Supermax ${ }^{\circ}$ (GT5) a que registrou maiores ocorrências no teste de inspeção visual, 18 luvas (9\%), resultado estatisticamente significante em relação às demais marcas $(\mathrm{p}<0,05)$. Nas 39 luvas que foram reprovadas no teste IV o defeito mais prevalente foi de regiões aderidas apresentando 23 luvas (58,9\%) com este defeito, sendo a região de dedos a predominante com 26 luvas (66\%).

As 968 luvas, aprovadas no teste IV, foram analisadas no teste de integridade (Tabela 2), destas 19 luvas (1,96\%) obtiveram escore 1 e 2 . O grupo GT1 (Embramac ${ }^{\circledR}$ ) apresentou os piores resultados, sendo estatisticamente significantes ( $\mathrm{p}$ $<0,05)$ em relação aos Grupos GT2, GT3 e GT5. Neste teste houve predomínio de vazamentos ou jatos que caracterizam os escores 1 e 2 na região dos dedos, 17 luvas (89,47\%).

A análise das 203 luvas contidas nas duas caixas marca EMBRAMAC ${ }^{\circledast}$ (GT1) resultou em uma porcentagem de 93,10\% (189 luvas) de luvas aprovadas para o uso, no entanto esta marca apresentou maior índice de luvas com escore 2 no teste de integridade, 7 luvas (3,53\%) das 198 analisadas. 
Das 200 luvas contidas nas duas caixas marca DESCARPACK ${ }^{\bullet}$ (GT2) a análise resultou em uma porcentagem de 97\% (194) de luvas aprovadas para o uso. Além dos defeitos estruturais, em especial, apresentou uma luva "sem colarinho" e outra com um inseto aderido ao látex na parte interna da luva, mais precisamente na região correspondente ao dedo indicador.

A análise das 202 luvas contidas nas duas caixas da marca SATARI ${ }^{\circ}$ (GT3) resultou em uma porcentagem de 98,02\% (198 luvas) de luvas aprovadas para o uso. Nas duas caixas da marca LEMGRUBER ${ }^{\circ}$ (GT4) havia um total de 200 luvas, a análise resultou em uma porcentagem de 94,50\% (189 luvas) de luvas aprovadas para o uso, no teste de inspeção visual esta marca apresentou alterações dos quatro tipos (manchas, regiões aderidas, rasgos ou furos). A marca SUPERMAX ${ }^{\circledR}$ (GT5) apresentou o pior desempenho, a análise das 200 luvas contidas nas duas caixas marca resultou em uma porcentagem de $89,00 \%$ (178 luvas) de luvas aprovadas para o uso. No teste de inspeção visual teve 18 luvas reprovadas (9\%), estas apresentavam manchas ou regiões aderidas; no teste de integridade 3 luvas $(1,6 \%)$ com escore acima de 0.

Com relação ao teste de hermeticidade, a marca EMBRAMAC ${ }^{\circledR}$ (GT1) apresentou o maior índice de luvas reprovadas (6,4\%), seguido pela marca LEMGRUBER ${ }^{\oplus}$ (GT4) com 3,46\%, SUPERMAX ${ }^{\circledast}$ (GT5) com 1,5\%, SATARI ${ }^{\circ}$ (GT3) com 0,99\% e DESCARPACK ${ }^{\circledast}$ (GT2) com índice de 0\% após o descarte de algumas luvas durante o teste de inspeção visual.

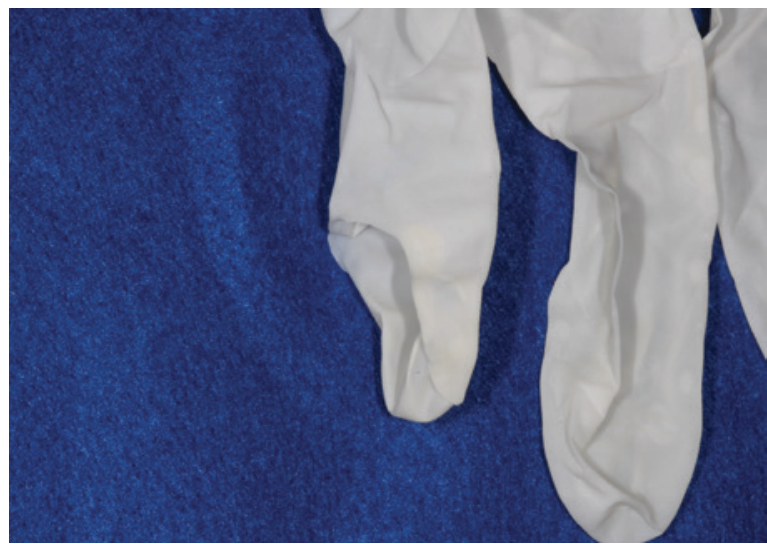

Figura 1. Luva reprovada no teste de inspeção visual por rasgo no dedo indicador

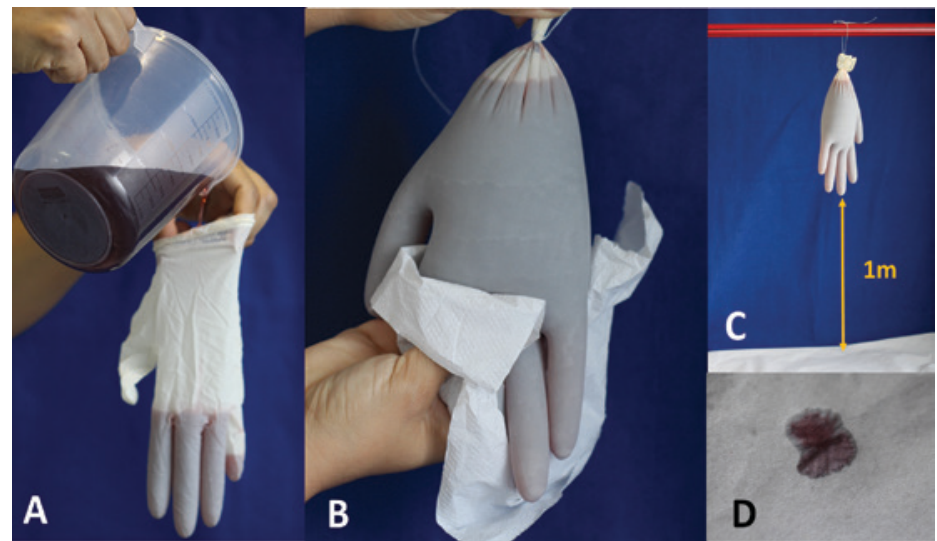

Figura 2. A - Inserção de $500 \mathrm{ml}$ de líquido evidenciador no interior da luva; B - Secagem da luva com papel absorvente; C - Luva posicionada a $1 \mathrm{~m}$ de camada de papel absorvente; $\mathrm{D}$ - Mancha no papel após vazamento em uma luva

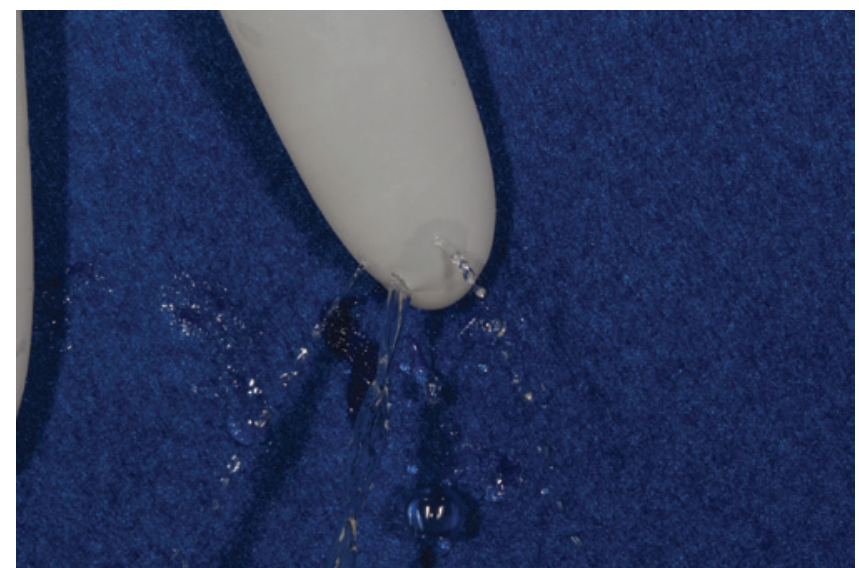

Figura 3. Luva com múltiplas perfurações sendo classificada no escore 2 para o teste de integridade 
Tabela 1. Porcentagem de luvas com defeito no Teste de Inspeção Visual (IV)

\begin{tabular}{|c|c|c|c|c|}
\hline Grupo-Teste & Total de luvas & $\mathbf{N}^{\circ}$ de luvas com defeito & Porcentagem de luvas com defeito (\%) & $\mathbf{P}^{*}$ \\
\hline GT1 & 203 & 5 & $2,46^{\text {ab }}$ & $<0,05$ \\
\hline GT2 & 200 & 6 & $3,0^{\text {ab }}$ & \\
\hline GT3 & 202 & 2 & $0,99^{\mathrm{a}}$ & \\
\hline GT4 & 202 & 8 & $3,96^{\mathrm{b}}$ & \\
\hline GT5 & 200 & 18 & $9,0^{\mathrm{c}}$ & \\
\hline TOTAL & 1007 & 39 & 3,87 & \\
\hline
\end{tabular}

Fonte: Coleta direta de dados na Clínica Odontológica da UFPI. *Letras minúsculas diferentes na mesma coluna significam diferença significativa pelo Teste Binomial para duas proporções $(P<0,05)$

Tabela 2. Comparação da porcentagem de luvas com defeito no Teste de Integridade (TI)

\begin{tabular}{|c|c|c|c|c|}
\hline Grupo-Teste & Total de luvas & $\mathrm{N}^{\circ}$ de luvas com defeito & Porcentagem de luvas com defeito (\%) & $P^{*}$ \\
\hline GT1 & 198 & 9 & $4,54^{a}$ & $<0,05$ \\
\hline GT2 & 195 & - & $0,0^{\mathrm{b}}$ & \\
\hline GT3 & 200 & 2 & $1,0^{b}$ & \\
\hline GT4 & 194 & 5 & $2,58^{\mathrm{ab}}$ & \\
\hline GT5 & 181 & 3 & $1,66^{b}$ & \\
\hline TOTAL & 968 & 19 & 1,96 & \\
\hline
\end{tabular}

Fonte: Coleta direta de dados na Clínica Odontológica da UFPI.*Letras minúsculas diferentes na mesma coluna significam diferença significativa pelo Teste Binomial para duas proporções $(P<0,05)$

Tabela 3. Resultados do teste de hermeticidade

\begin{tabular}{|c|c|c|c|c|}
\hline Grupo-Teste & Luvas com furos ou rasgos no teste IV & Luvas com escore $\mathbf{1}$ e $\mathbf{2}$ no teste TI & \multicolumn{3}{|c|}{ Luvas reprovadas no teste de hermeticidade } \\
\hline & & & N & 13 \\
\hline GT1 & 4 & 9 & - & 6,40 \\
\hline GT2 & - & - & 2 & 0,99 \\
\hline GT3 & - & 2 & 7 & 3,46 \\
\hline GT4 & 2 & 5 & 3 & 1,50 \\
\hline GT5 & - & 3 & \\
\hline
\end{tabular}

Fonte: Coleta direta de dados na Clínica Odontológica da UFPI

\section{Discussão}

As luvas de látex, juntamente com gorros e máscaras são os principais equipamentos de proteção individual dos profissionais de saúde. Dessa maneira, esses equipamentos devem ter um alto controle de qualidade na sua fabricação para promover uma proteção efetiva.

As normas do INMETRO (Instituto Nacional de Metrologia, Qualidade e Tecnologia) fixam os requisitos exigíveis de luvas de procedimento não cirúrgico e luvas cirúrgicas; com a finalidade de se verificar a efetividade do produto com relação ao desempenho, são aplicados diversos testes, dentre eles o de hermeticidade, uma luva sem colarinho como a encontrada nas caixas da marca DESCARPACK ${ }^{\oplus}$ provavelmente não garante o vedamento adequado na região do punho sendo incapaz de garantir uma adequada hermeticidade. ${ }^{17}$

O trabalho apresentou resultados semelhantes ao de Serratine et al. (2007) que demonstram que há variação de qualidade entre as marcas, concluindo que embora todas as luvas avaliadas apresentassem variações morfológicas, algumas apresentavam somente depressões, enquanto outras apresentavam áreas de fragilidade propícias às perfurações. ${ }^{7}$

O INMETRO determina um nível de qualidade aceitável (máximo percentual de unidades defeituosas para fins de inspeção de amostragem) esse valor corresponde a 1,5\% das luvas nos testes de hermeticidade (presença de furos) para um nível de inspeção (relação entre o tamanho do lote e o tamanho da amostra) do tipo S4 (tipo de nível de inspeção especial que é usado quando forem necessários tamanhos de amostra relativamente pequeno) semelhante ao que foi realizado no presente 
estudo, que utilizou aproximadamente 200 luvas em um universo de 500.000 que estão presentes em um lote. ${ }^{17}$

No presente estudo, luvas reprovadas quanto a hermeticidade (Tabela 3) são caracterizadas como as que apresentaram rasgos ou furos no Teste IV e as que apresentaram escores 1 ou 2 no Teste TI. No quesito hermeticidade, as marcas comerciais EMBRAMAC ${ }^{\circledast}$ e LEMGRUBER ${ }^{\circledR}$ foram reprovadas e a marca SUPERMAX ${ }^{\star}$ obteve um resultado no limite imposto pelo INMETRO. As marcas aprovadas foram a DESCARPACK ${ }^{\oplus}$ e SATARI ${ }^{\bullet}$.

Os defeitos estruturais, como o de manchas e regiões aderidas avaliadas no primeiro teste, têm importância, já que são áreas de fragilidade ou deficiência do látex, extremamente susceptíveis a ocorrência de furos ou rasgos comprometendo a hermeticidade dessas luvas.

\section{Conclusão}

Todas as marcas comerciais das luvas de procedimentos que foram testadas apresentaram perfurações. Dentre as marcas comerciais avaliadas, em termos percentuais, no teste IV a marca SATARI ${ }^{\circledR}$ obteve o melhor desempenho, enquanto que a SUPERMAX ${ }^{\circledR}$ ficou com o pior resultado. Já no teste TI, o melhor desempenho ficou com a marca DESCARPACK ${ }^{\circledast}$ o pior resultado foi o da marca EMBRAMAC ${ }^{\bullet}$. No quesito hermeticidade, em comparação com o nível de qualidade aceitável, imposto pelo INMETRO, as marcas EMBRAMAC ${ }^{\circledR}$ e LEMGRUBER ${ }^{\circledR}$ foram reprovadas, a marca SUPERMAX ${ }^{\circledR}$ ficou no limite e as marcas DESCARPACK ${ }^{\bullet}$ SATARI $^{\circ}$ foram aprovadas.

\section{Referências ::}

1. Cavalli LS, Karam FSC, de Brito KCT, de Brito BG. Existe relação entre ética e biossegurança ocupacional? Revista Contraponto. 2015;1(3):176-80. 2. Tomo S, Lima DP, Correia ASC. Conhecimento de graduandos em Odontologia a respeito das normas de biossegurança. Arch Health Invest. 2014;3(4):9-17.

3. Samaranayake LP, Sheutz F, Cottone JA. Controle da infecção para a equipe odontológica. 2nd ed. São Paulo: Santos; 1993, p. 146.

4. Lopes N, Prates N, Rabelo R, Cruz JFW. Análise da permeabilidade das luvas de látex para procedimento mais utilizadas por alunos da $\mathrm{Fa}$ culdade de Odontologia da Universidade Federal da Bahia. R. Ci. Med. biol. 2009;8(2):206-12.

5. Cohen JVFB, Leão MVP, dos Santos FFS. Condutas de biossegurança relacionadas aos trabalhos protéticos utilizadas por cirurgiones-dentistas de Porto Velho (RO). Rev. Bras. Odontol. 2013;70(1):93-6.

6. Lucena VCF, de Morais HHA, Dias TGS, Barbalho JCM. Avaliação da integridade das luvas cirúrgicas e de procedimentos após atendimentos odontológicos. Rev. Cir. Traumatol. Buco-Maxilo-Fac. 2013;13(3): 71-8.

7. Serratine ACP, Pacheco E, Miero M. Avaliação da integridade das luvas cirúrgicas após a utilização em cirurgias odontológicas. Arquivos Catarinenses de Medicina. 2007;36(1):85-9.

8. Cavalcanti AL, Costa LB, Dantas OAB, Melo ES. Avaliação da integridade de luvas de procedimentos após atendimento odontológico. POS - Perspect. Oral Sci. 2010;2(2):15-9.

9.Kuroyanagi N, Nagao T, Sakuma H, Miyachi S, Ochiai Y, Kimura H, et al. Risk of surgical glove perforation in oral and maxillofacial surgery. Int J Oral Maxillofac Surg. 2012;41(8):1014-9.

10. Pitten FA, Herdemann G, Kramer A. The integrity of latex gloves in clinical dental practice. Infection. 2000;28(6):388-92.

11. Korniewicz DM, Garzon L, Plitcha S. Health care workers: risk factors for nonlatex and latex gloves during surgery. AIHA J (Fairfax, Va). 2003;64(6):851-5.

12. Korniewicz DM, Garzon L, Seltzer J. Failure rates in nonlatex surgical gloves. Am J Infect Control. 2004;32(5):268-73

13. Machado E, Flores FW, Barbieri S, Frantz E, Flores JA. Avaliação dos testes empregados para detecção de perfurações em luvas cirúrgicas. Arquivos Catarinenses de Medicina. 2008;37(3):34-8.

14. Phalen RN, Wong WK. Integrity of disposable nitrile exam gloves exposed to simulated movement. J Occup Environ Hyg. 2011;8(5):289-99.

15. Otis LL, Cottone LA. Prevalence of perfurations disposable latex gloves during routine dental treatment. Journal of the American Dental Association. 1989;118(3):321-4.

16. Teixeira AR, Fernandes RA, Serratine ACP. Perfurações em luvas de látex utilizadas em cirurgias odontológicas. Odontologia. Clín.-Científ. 2008;7(2):145-50.

17. BRASIL. (2012) Ministério do Desenvolvimento, Indústria e Comércio Exterior. Instituto Nacional de Metrologia, Qualidade e Tecnologia-INMETRO. Portaria n. ${ }^{\circ}$ 451, de 31 de agosto de 2012. Brasília, p. 1-3.

Recebido em: 19/04/2016 / Aprovado em: 08/05/2016

Alessandro Ribeiro Gonçalves

BR 343, n 6080, casa 12, Condomínio Villa Inglesa, Bairro Gurupi Teresina/PI, Brasil - CEP: 64091-210

E-mail: argoncalves@yahoo.com 